\section{Anåsthesie und Organprotektion Nur Mittel zum Zweck (nicht weiter zu schädigen) oder gar „Heilschlaf“?}

\author{
Norbert Roewer • Peter Kranke
}

Nur Mittel zum Zweck (nicht zu

schädigen) oder gar „Heilschlaf“?

Seite 542

Perioperative Myokardprotektion

bei nicht kardiochirurg. Eingriffen Seite 546

Einfluss des anästhesiolog.

Managements auf die intra-

operative Neuroprotektion

Seite 556

Nephroprotektion: anästhesiolog.

Management bei Nieren-

transplantation

Seite 566
Als der Bericht von Henry Jacob Bigelow über die „Insensibility during surgical operations produced by inhalation" am 18.11.1846 im Boston Medical and Surgical Journal (heute: N Engl J Med) erschien, stand die Empfindungslosigkeit („Insensibility“) als Novum ganz im Vordergrund. Und damit verbunden die Verwunderung darüber, dass gewollt und zeitlich begrenzt das Bewusstsein derart beeinflusst werden konnte, dass eine chirurgische Prozedur - zunächst eine Tumorentfernung am Kiefer bzw. Hals - ohne die sonst übliche drastische Schmerzäußerung durchgeführt werden konnte [1].

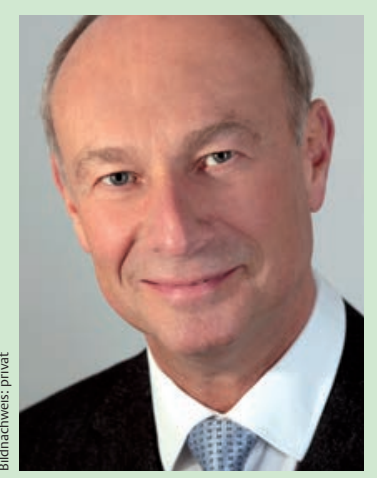

Univ.-Prof. Dr. Dr. h.c. Norbert Roewer ist Direktor und Ordinarius an der Klinik und Poliklinik für Anästhesiologie des Universitätsklinikums Würzburg und stellvertretender ärztlicher Direktor des Universitätsklinikums Würzburg. Er ist Mitglied im engeren Präsidium der DGAl. Prof. Roewer gehört zum Herausgeber-Team der AINS.E-Mail:AN_Direktion@ ukw.de
Chirurgie ohne Schmerz Die Einführung des Chloroforms im Jahre 1847 durch James Young Simpson zu Narkosezwecken sowie auch dessen Einsatz bei der geburtshilflichen Anästhesie bei Königin Victoria am 7. April 1853 anlässlich der Geburt von Prinz Leopold durch John Snow war von dem Wunsch geprägt, Abhilfe gegen - bis dato unerträgliches - Leid im Rahmen eines chirurgischen Eingriffs bzw. während der Geburt zu schaffen.

Neue Möglichkeiten Danach folgten die Pionierzeiten mit der ersten Durchführung einer Kaiserschnittentbindung am 25. Januar 1847, der ersten Appendektomie (27. April 1887), ersten Anwendungen der Spinalanästhesie durch August Bier (16. August 1897) und der (spinalen) geburtshilflichen Analgesie in Basel durch Oskar Kreis im Jahre 1899 sowie mit zahlreichen pharmakologischen Entwicklungen [2]. Diese Zeiten waren mehr oder minder allesamt geprägt von der zunehmenden Realisierbarkeit chirurgischer Eingriffe - zunächst einmal generell und später auch vermehrt bei Patienten mit Vorerkrankungen und Leistungseinschränkungen.

Naturgemäß erschienen bald auch erste Berichte von Zwischenfällen im Kontext der Chloroform-

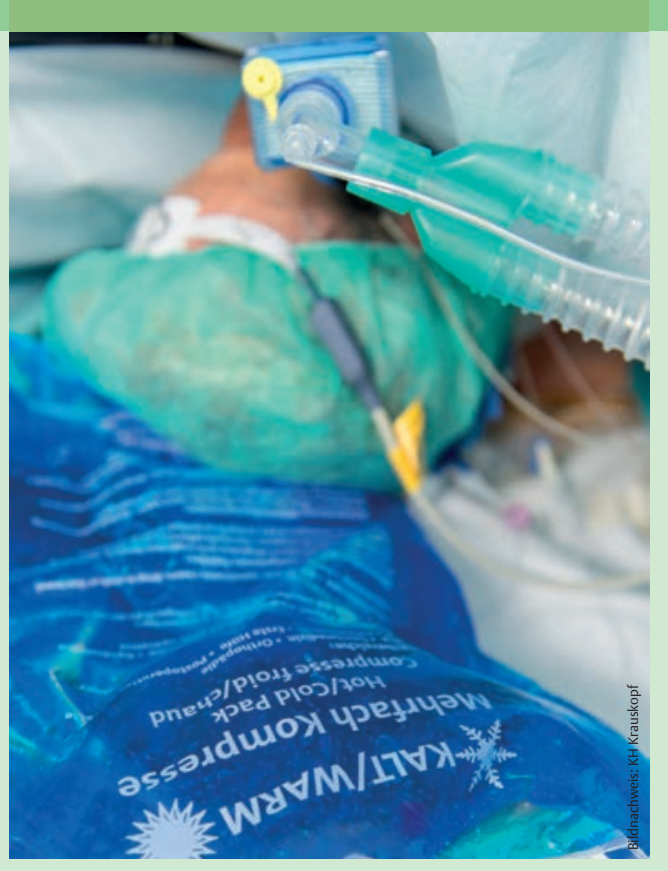

oder Lachgasanalgesie (1848 bzw. 1873). Doch im Großen und Ganzen kannte die Entwicklung nur eine Richtung:

- Eingriffe wurden zunächst überhaupt möglich und waren später mit zunehmend geringerer Morbidität und Letalität assoziiert.

Entwicklung der anästhesiebezogenen Letalität Eine im Jahr 2012 publizierte Zusammenschau der perioperativen und anästhesiebezogenen Letalität kommt im Rahmen der metaanalytischen Aufarbeitung von 87 Studien an insgesamt 21,4 Mio. Anästhesien zu einer auf die Anästhesie bezogenen Letalität von

- 357/1 Mio. für den Zeitraum vor 1970,

- 52/1 Mio. im Zeitraum 1970-1980 und

- 34/1 Mio. für den Zeitraum 1990-2000.

Derzeit wird - basierend auf einer anderen Datenbasis - von etwa 8,2 anästhesiebedingten Todesfällen pro 1 Mio. Krankenhausentlassungen ausgegangen. Naturgemäß gibt es verfahrensimmanente Unsicherheiten in der jeweiligen Abschätzung [3].

Rückgang der perioperativen Mortalität Gleichzeitig zeigt sich ein Rückgang der perioperativen Mortalität 
- von 10603/1 Mio. (vor 1970),

- über 4533/1 Mio. (1970-1980)

- hin zu 1176/1 Mio. (1990-2000).

Daraus schlussfolgern die analysierenden Autoren, dass trotz des gestiegenen Ausgangsrisikos die perioperative Mortalität drastisch sinkt. Es zeigen sich hierbei ausgeprägte regionale Disparitäten und der positive Trend ist nachvollziehbarer Weise besonders stark in den sog. „entwickelten Ländern“ mit hohem Human Development Index (HDI) zu verzeichnen [4].

Auch eine Frage der Relation Betrachtet man die Mortalitätsraten, darf dabei gleichwohl die Exposition(-swahrscheinlichkeit) nicht außer Acht gelassen werden. Zwar relativiert die niedrige Wahrscheinlichkeit eines Ereignisses das Bedrohungspotenzial für den individuellen Patienten.

- Berücksichtigt man jedoch auch die Expositionswahrscheinlichkeit für einen chirurgischen Eingriff, sind die absoluten Zahlen nicht unerheblich.

So wurde für WHO-Mitgliedsstaaten mit hohen Gesundheitsausgaben (> 1000 US-Dollar pro Kopf und Jahr) eine Rate von 11110 Eingriffen pro 100000 Einwohner pro Jahr berechnet [5]. Somit unterzieht sich in etwa ein Zehntel der dortigen Populationen jährlich einem operativen Eingriff. Bezogen auf die oben dargelegte perioperative Mortalität (1176/1 Mio.) ist die auf diese Weise approximierte perioperative Mortalität nicht unwesentlich.

Weiteres Beispiel In eine ähnliche Richtung weisen die Daten der „European Surgical Outcomes Study“ (EuSOS), einer 7 Tage laufenden Kohortenstudie der European Society of Intensive Care Medicine und der European Society of Anaesthesiology aus dem Jahre 2011 [6]. In dieser Kohorte >16-jähriger, nicht kardiochirurgischer Patienten aus 28 europäischen Ländern (498 Krankenhäuser) wurden die Patienten für max. 60 Tage u.a. mit Blick auf den primären Endpunkt „in-hospital mortality“ beobachtet.

Von den eingeschlossenen und analysierten 46539 Patienten starben 1855 (4\%) vor Entlassung aus dem Krankenhaus. Von den aus Deutschland beigesteuerten 5284 Patienten verstarben 133, was einer Sterblichkeit vor Entlassung von 2,5\% entspricht.

Wenn die Autoren schlussfolgern, dass die Sterblichkeit dieser nicht kardiochirurgischen Patienten höher als erwartet lag, mag dies vielen Aspekten zuzuschreiben sein, auf die hier nicht weiter eingegangen werden soll.

Kein unerheblicher Faktor Alle vorgebrachten Daten scheinen aber eines zu belegen:

- Perioperative Letalität ist national und global ein nicht unerheblicher Faktor

- auch wenn für bestimmte (elektive) Eingriffe in Hinblick auf die perioperative und anästhesiolo- gisch bedingte Sterblichkeit objektiv weder Sorge noch Angst begründet sein mögen und sich die Zahlen in den letzten Dekaden insgesamt zum Positiven entwickelt haben. Bartels und Mitautoren [7] listeten die „Sterblichkeit im Zeitraum von 30 Tagen nach einem chirurgischen Eingriff" gar in der Sequenz der häufigsten Todesfälle noch vor der Anzahl der Todesfälle aufgrund zerebrovaskulärer Erkrankungen - basierend auf Daten der Centers for Disease Control (CDC) und der „Nationwide Inpatient Sample“-Datenbank aus den USA (๑ Abb. 1).

Rolle der Anästhesie Die Reduktion perioperativer Letalität über die Zeit ist mannigfaltigen Bemühungen zur Verbesserung des perioperativen Outcomes geschuldet: Anstrengungen im Hinblick auf die Sterblichkeit an sich, aber auch auf die Reduktion zahlreicher Endpunkte zu den verschiedensten Aspekten perioperativer Organ(dys)funktion. Nicht zuletzt vor diesem Hintergrund und aufgrund vielversprechender experimenteller Untersuchungen (Stichwort: Anästhetika induzierte Präkonditionierung) sind anästhesiologische Maßnahmen bzw. die anästhesiologische Pharmakotherapie mitunter phasenweise zu einem bedeutsamen Werkzeug einer besseren perioperativen Protektion avanciert.

Topthema Wie weit sich diese Hoffnung zur günstigen Beeinflussung von Organfunktion und Outcome bewahrheitet haben und welche Maßnahmen - etabliert oder experimentell - vielversprechend in Hinblick auf ein besseres Überstehen eines chirurgischen Eingriffs bzw. der Organdysfunktion sind, ist Gegenstand dieses Topthemas. Dabei soll nicht ausschließlich auf spezifische Maßnahmen abgezielt werden. Vielmehr soll der Blick über den Tellerrand gewagt werden, welche Interventionen (inkl. der Diagnostik) nützlich für Herz, Hirn, Niere und das Überleben („Health“) generell sind.

\section{Ausmaß der perioperativen Sterblichkeit}

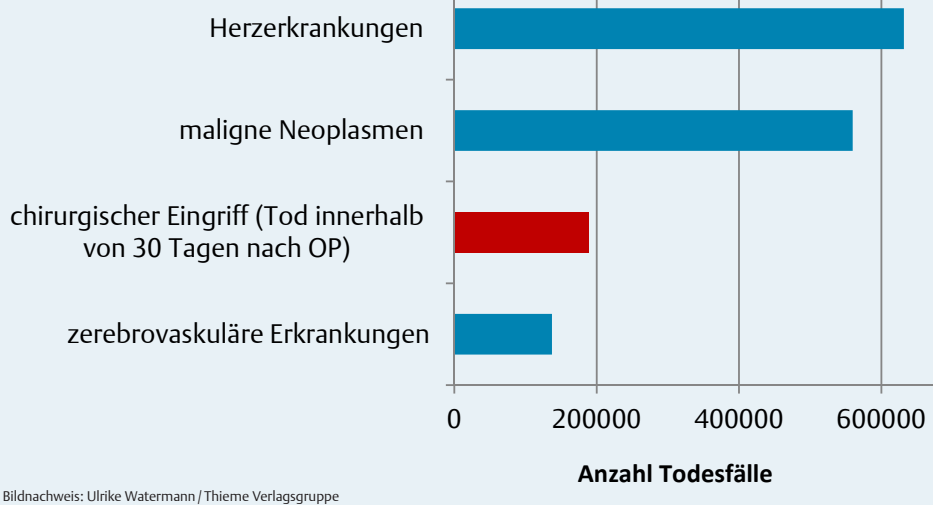


Myokardprotektion Stumpner und Kollegen beschreiben im ersten Beitrag, welche Maßnahmen beim kardial vorerkrankten Patienten vielversprechend sind. Sie „demystifizieren“ andererseits aber auch Interventionen, denen - z. T. trotz vielversprechender experimenteller Nachweise ein wirklich gut belegter positiver Effekt auf Morbidität und perioperative Letalität schlussendlich nicht mit der erforderlichen Sicherheit attestiert werden konnte.

Neuroprotektion Im Beitrag von Serge Thal und Anne Sebastiani steht das Erfolgsorgan allgemeinanästhesiologischer Bemühungen im Fokus: das Gehirn und seine Erkrankungen. Insbesondere wird der relevanten Frage nachgegangen, wie anlässlich möglicherweise aufgehobener Autoregulation, bereits vorbestehender kognitiver Defizite oder stattgehabter zerebrovaskulärer Erkrankungen Anästhesie im weitesten Sinne sicher und ohne zusätzliche negative Beeinträchtigung der kognitiven Leistungsfähigkeit durchgeführt werden kann und sollte.

Nephroprotektion bei Nierentransplantation Smul und Kollegen thematisieren abschließend das anästhesiologische Management der Nierentransplantation und die Strategien der Nephroprotektion im Allgemeinen. Sie gehen dabei insbesondere auf die Reduktion des IschämieReperfusionsschadens, das optimale Management der Transplantatperfusion, die Meidung nephrotoxischer Substanzen und eine effektive Immunsuppression im Zuge der perioperativen Therapie sowie auch der Nachsorge der Patienten ein.

Allgemein- vs. Regionalanästhesie Herausgefordert werden die Verheißungen allgemeinanästhesiologischer Interventionen in Hinblick auf eine positive Beeinflussung der perioperativen Letalität gleichwohl von dem Umstand, dass gerade regionalanästhesiologische Verfahren nicht selten mit verbessertem perioperativen Outcome in Zusammenhang gebracht werden [8, 9]. Ein Umstand, der ebenso wie die medikamentöse und anästhesiologische Präkonditionierung oft im Kreuzfeuer der Debatten steht [10] und der generell und für viele Patientenkollektive bzw. spezifische Interventionen zum jetzigen Zeitpunkt keinesfalls schlüssig beantwortet ist [11]. Und so betitelte das Journal Anesthesia \& Analgesia das Cover seiner September-Ausgabe 2014 zum dargestellten „Kampf“ der Regionalanästhesie („Nadel“) mit dem Verfahren der Allgemeinanästhesie („Blade“) treffend mit den Worten „Is the needle truly mightier than the blade?“.

Inwieweit besagte regionalanästhesiologische Effekte ebenso wie spezifische Maßnahmen im Kontext einer Allgemeinanästhesie zur Organprotektion jemals für allgemeine Patientenkollektive in ihrer Wertigkeit einzugrenzen sind, mag zu Recht bezweifelt werden. Vielleicht sollte dies auch nicht Ziel der Forschungs- und Versorgungsbemühungen sein, sondern vielmehr nachteilige Konstellationen und Pathomechanismen dingfest zu machen und tunlichst zu minimieren.

Praktizieren und Studieren Nicht zuletzt ist ein Aspekt nicht unerheblich: die tägliche Übung und die Versiertheit der Akteure durch tägliches Praktizieren im Alltag. Einflussvariablen, die sich in klinischen Studien wie auch Untersuchungen an großen Kohorten oft nur unzureichend abbilden lassen.

Die Erfahrung lässt sich zwar nicht erlesen, gleichwohl aber das theoretische Rüstzeug zur guten Praxis. Gewappnet mit dem Hintergrundwissen aus den folgenden Beiträgen und dem Wissen um Referenzwerke zur perioperativen Konditionierung, auf die in den Beiträgen verwiesen wird, sollte dann auch die Durchführung der Anästhesie bei kardio- und zerebrovaskulär oder renal vorerkrankten Patienten kein unüberwindbares Problem in der klinischen Versorgung mehr sein.

In diesem Sinne wünschen wir Ihnen eine erkenntnisreiche und unterhaltsame Lektüre und natürlich stets ein gutes Ergebnis Ihrer Arbeit!

Ihre Norbert Roewer und Peter Kranke

\section{Literaturverzeichnis}

1 Bigelow HJ. Insensibility during surgical operations produced by inhalation. Boston Med Surg J 1846; 35: 309-331

2 History of Anaesthesia Society. Timeline of important dates and events in the development of anaesthesia. Im Internet: http://www.histansoc.org.uk/timeline.htmI (Stand: Mai 2015)

$3 \mathrm{Li} \mathrm{G}$, Warner M, Lang BH et al. Epidemiology of anesthesiarelated mortality in the United States, 1999-2005. Anesthesiology 2009; 110: 759-765

4 United Nations Development Programme. Human Development Report 2010 - $20^{\text {th }}$ Anniversary Edition. New York: United Nations Development Program; 2010

5 Weiser TG, Regenbogen SE, Thompson KD et al. An estimation of the global volume of surgery: a modelling strategy based on available data. Lancet 2008; 372: 139-144

6 Pearse RM, Moreno RP, Bauer P et al. Mortality after surgery in Europe: a 7 day cohort study. Lancet 2012; 380: 1059-1065

7 Bartels K, Sullivan BL, Eltzschig HK. TnT: blowing the cover from perioperative myocardial injury. Anesthesiology 2014; 120: 533-535

8 Guay J, Choi PT, Suresh S et al. Neuraxial anesthesia for the prevention of postoperative mortality and major morbidity: an overview of cochrane systematic reviews. Anesth Analg 2014; 119: 716-725

9 Guay J, Choi P, Suresh S et al. Neuraxial blockade for the prevention of postoperative mortality and major morbidity: an overview of cochrane systematic reviews. Cochrane Database Syst Rev 2014; 1: CD010108; DOI: 10.1002/14651858.CD010108.pub2

10 Memtsoudis SG, Liu SS. Do neuraxial techniques affect perioperative outcomes? The story of vantage points and number games. Anesth Analg 2014; 119: 501-502

11 Kooij FO, Schlack WS, Preckel B, Hollmann MW. Does regional analgesia for major surgery improve outcome? Focus on epidural analgesia. Anesth Analg 2014 ; 119: 740-744 unter http://dx.doi.org/

10.1055/s-0041-103105 